\title{
Skid adjuster for humps
}

\author{
Alexander Demyanov ${ }^{1}$, Alexey Demyanov ${ }^{1 *}$, and Alexander Rybak ${ }^{1}$ \\ ${ }^{1}$ Don State Technical University, Gagarin sq., 1, Rostov-on-Don, 344000, Russia
}

\begin{abstract}
The paper based on the analysis of the working conditions of railway transport shows that, ceteris paribus, an increase in actual durability and mean time before failure of rolling stock units is obtained through maintenance of wheelsets, especially during logistic processes on nonmechanized humps. One of the main causes for the service life decrease of wheelsets is brake damage. To eliminate such brake damage on highways, on-board braking dis-tance regulators mounted on the locomotive are used. In the hump yards, using such systems is not possible, since under marshalling, cars are detached from the locomotive. In this regard, an industrial challenge turns up - to design a similar in purpose braking distance regulator for humps through skidding. To solve this problem, we developed a method of controlling the speed of cars to be sorted, which complements the widely used technology of the shoe braking and avoids the formation of one-way sliders. The paper presents a skid adjuster, which excludes brake damage to the rolling stock wheels by the slides on the humps using the shoe braking techniques. The motion equation of the rolling stock equipped with a skid adjuster on the hump tracks is developed. In the result of this study, an equation of car motion in the system of "rolling stock - Hughes regulator - way" allowing to enter the working elements of Hughes controller to an existing and debugged algorithm of any slides with minimal change it.
\end{abstract}

\section{Introduction}

For Russia, railway transport plays one of the key and strategic parts, as it is the main transport artery connecting all its regions. The efficient operation of this artery deter-mines largely the state of the country's economy and security. The efficiency and competitiveness of the railway transport is largely owing to increasing speed and volume of cargo transportation, as well as to improving reliability of a new rolling stock and life support of the operated rolling stock $[1,2,13]$. Ceteris paribus, an im-provement of the actual durability and mean time before failure of the crews is achieved through the maintenance of the rolling stock wheels under actual operating conditions including the logistic processes in the hump yards $[3,5,6]$.

One of the key reasons for reducing the service life of wheelsets is brake damage under wheel sliding, which is called skidding. One of the most common and danger-ous types of brake damage is a slide - a straight segment on the wheel surface formed under thermomechanical processes. The operation of wheels with slides causes breakdown of the

\footnotetext{
*Corresponding author: alexys61@yandex.ru
} 
elements of the upper structure of the track, car detention for wheelset change, costs for restoring or manufacturing of new wheels, etc. $[3,5,6]$.

To eliminate such brake damage, on-board braking distance regulators mounted on a locomotive are used on highways [7]. In the hump yards, application of this system is not possible, since under marshalling, cars are detached from the locomotive. In this regard, an industrial challenge is encountered - to design a similar in purpose braking distance adjuster for humps through skidding.

Currently, marshalling on humps is carried out using beam or skate retarders. At this, the braking system for each hump yard is selected individually according to the ratio of cargo turnover and operating costs for retarders. So, if beam retarders are effective in the high-powered hump yards (over 30 tracks or more than 3,500-5,500 cars per day); then skate retarders (SR) are widely used in the hump yards of low and medium power (up to 1,500 and up to 3,000 cars per day, respectively). In addition, industrial enterprises have humps with skate braking.

The principle of skate braking for such humps is most adequate for costs reasons [8]. At this, a major drawback of the skate braking technique is the possibility of thermomechanical damage to the surface of the wheels, which, on the one hand, causes losses to downtime of cars during the inspection and change of wheelsets, and on the other hand, the expenditures on restoring or manufacturing of new wheels. Thermomechanical damage to the wheels on the humps is called one-sided slides.

The elimination of this negative phenomenon complete with the minimum material and capital intensity of the skate braking principle offers a challenge for saving large material resources of the industry [8].

\section{Operating Principle and Methods}

According to the task, a rate control technique for sorted cars complementing a widely used skate -braking technology and eliminating the formation of one-sided slides is developed. The technique is based on the alteration of the wheels - rails contact surface due to the periodic transition of the padded set from sliding friction to rolling friction. To implement this technique, a stationary skid length adjuster (anti-slide safety system) which is an ordered system of devices (working modules) located on the neck of the track rail on the side of the brake shoe in a predetermined order with a certain interval is developed [9-11].

According to the methodology, the entire slidepath of the wheelset (LT) at each brake position is divided into intervals ( $\mathrm{Li}$ ), within which the formation of slides on the wheels is excluded:

$$
L_{T}=\sum L_{i}
$$

Each working module of the anti-slide safety system includes a wedge element with two inclined faces.

The transition of the wheelset from sliding to rolling is carried out through cresting of the padded wheel flange onto the working module, its rising over the brake skate and turning of the whole set to a preset angle. As a result of turning, a friction section of the non-padded wheel with the rail is changing until damage is formed on it.

Since the principle of operation is based on the module - wheel interaction, then, being a new element built into the existing path, the modules of the anti-slide safety system should fit into the existing characteristics of the path. The case in hand is about the dynamic interaction of the rolling stock wheels and modules of the anti-slide safety system, and it is obvious that the value of wheel impact on a new track element should be at the level of 
collisions with typical track elements such as, for example, a rail joint, etc.

To describe the rolling stock movement, equations are used that relate the driving force to the resistance forces occurring under the movement [12]:

$$
m \frac{d^{2} x_{i}}{d t}=m \frac{d^{2} x_{0}}{d t}-\sum_{i=1}^{i=n} F_{i} \quad \text { or } \quad m a_{i}=m a_{0}-\sum_{i=1}^{i=n} F_{i}
$$

where $\mathrm{m}$ is weight of the rolling stock; $\mathrm{a}$ is acceleration of the rolling stock movement; $\Sigma \mathrm{Fi}$ is the sum of the resistance forces; $F=$ ma is driving force.

\section{Research Results}

Cyclograms of the car movement under the splitting up of trains along the hump yard are developed. On the basis of the made cyclograms, the driving conditions are determined considering the wheelsets - skid adjuster elements contact. The resulting equation is a complete equation of the rolling stock movement for these conditions, and, therefore, using it, it is possible to calculate the magnitude of the driving force and resistance forces at any stage of the movement with account for the design parameters of the modules of the antislide safety system.

Obviously, to compile the equation of the car movement in the hump yard with account for friction, one can use the equations (2). In this case, if at the initial moment of motion, the driving force is equal to F0, the magnitude of the driving force at the i-th moment of time is respectively $\mathrm{Fi}$, and the friction forces acting during the movement are equal to FTp, then the equation (3) can be presented in the following form:

$$
F_{0}-F_{i}=F_{m p} \text {. }
$$

If the acceleration at the initial movement time is a0, and at the i-th movement point, it is respectively ai, then the equation (3) on transforming it with respect to mai, can already be presented as

$$
m a_{i}=m a_{0}-F_{m p}
$$

The cycle of wheelset movement along the hump yard equipped with the anti-slide safety system can be divided into five stages (Fig. 1). The cyclogram of the car movement stages implies that:

$$
T=T_{1}+T_{2}+T_{3}+T_{4}+T_{5}, \quad \text { or } \quad T=\sum_{i=1}^{i=5} T_{i} .
$$

The first stage (T1). The wheelset is rolling along the rails on the tread surface. In the wheel - rail contact, the rolling friction force Fктрк осcurs.

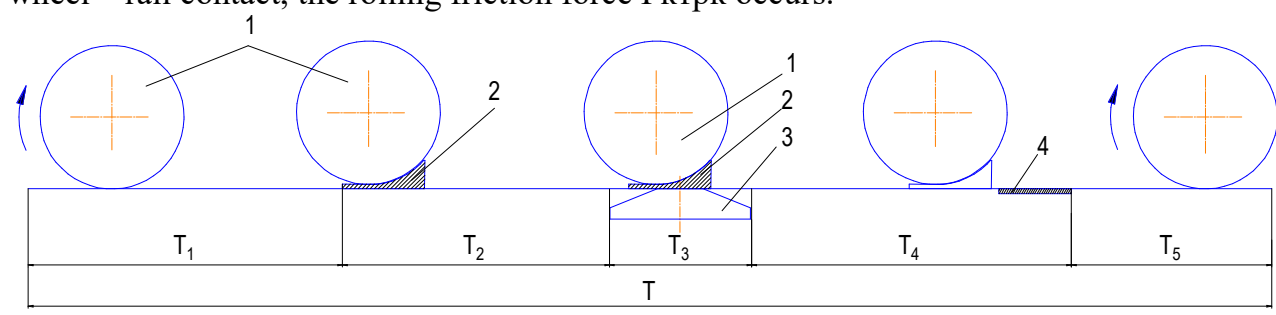

Fig. 1. Cyclogram of car movement in hump yard 1 is wheel, 2 is skate brake, 3 is working module, 4 is derailer

We write the equation (5) for this stage considering the description of the contact 
features

$$
m a_{1}=m a_{0}-F_{m p c}^{\kappa} .
$$

Let $\mathrm{fk}$ be the rolling friction coefficient, and $\mathrm{G}-$ the axial load distributed on one wheel, then for a wheelset:

$$
m a_{1}=m a_{0}-2 G f_{\kappa} .
$$

The second stage (T2). At this stage, a skate brake is installed under the wheel of a wheelset, and skidding starts. If the sliding friction force on the rolling surface is Fктрс, and the sliding friction coefficient is $\mathrm{fc}$, then

$$
m a_{2}=m a_{0}-m a_{1}-2 F_{m p c}^{\kappa}, \quad \text { or } \quad m a_{2}=m a_{0}-2 G \cdot f_{\kappa}-2 G \cdot f_{c} .
$$

The third stage (T3). Under skidding, the padded wheel is crested onto the module of the anti-slide safety system and, rising above the shoe, starts rolling along the level section. This stage is in turn divided into three parts (Fig. 2):

$$
T_{3}=T_{3.1}+T_{3.2}+T_{3.3}
$$

where T3.1 - in this section, the padded wheel comes into contact with an inclined face of the module, and the transition from the sliding friction of the wheel along the tread surface to the rolling friction of the wheel flange modulo begins. Here, at the moment of transition, the padded wheel tread simultaneously contacts with the skate, and the radial surface of the wheel flange - with the inclined wedge face.

Consider the beginning of the wheel - wedge contact and the conditions for the transition from friction-sliding to rolling friction (Fig. 3, a). At the moment of the contact, at some point $\mathrm{A}$ from the driving force $\mathrm{F}$, a flange impact upon a face caused by the force F1 occurs. The analysis of the shock loads of the wheel on the elements of the upper structure of the track is customary [3] to perform according to the formula:

$$
F_{1}=\alpha \cdot V \sqrt{C_{r} \cdot m_{\kappa n}}
$$

where $\mathrm{V}$ is rolling stock speed, $\alpha$ is attack angle of the wheel on the rail, ткп is weight of the wheelset and its unsprung parts rigidly bound with it, cr is the stiffness of the rail wheel system in the horizontal direction transverse to the track centre.

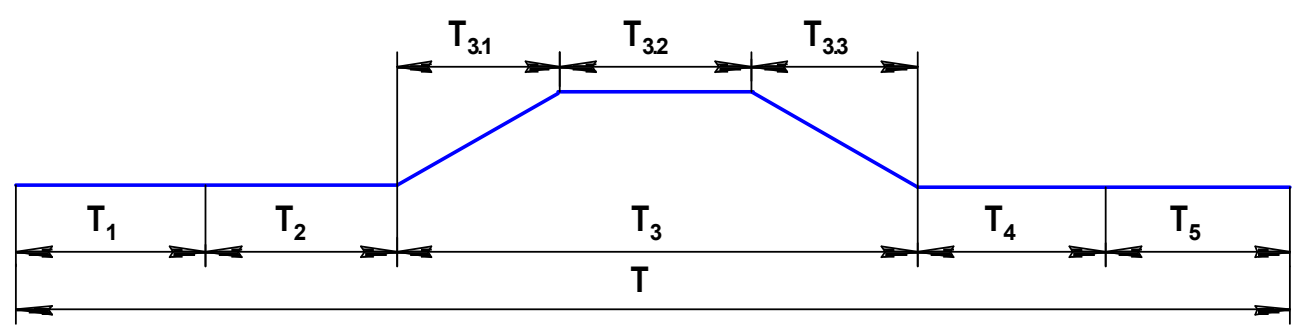

Fig. 2. Cyclogram of car movement in hump yard considering periods of contact between wheelset and anti-slide safety system module

T1 is wheelset rolling, T2 is car movement on shoe, T3.1 is wheel tripping-over on inclined module face, T3.2 is movement on wheel flange modulo, T3.3 is rolling-down of flange from module, T4 is car movement on shoe, T5 is wheelset rolling

This force at point A decomposes into the normal force Fn and the force F2 of the movement along the face. Normal Fn can be expressed in terms of G, and the driving force $\mathrm{F} 2$ can be expressed in terms of F1. 


$$
F_{n}=\frac{G}{\cos \alpha}, \quad F_{2}=\frac{F_{1}}{\cos \alpha} .
$$

At point B from the axial load $\mathrm{G}$ fallen upon the wheel on the tread surface and the rail, the sliding friction force Fктрс of the non-padded and padded wheels will also occur, i.e.

$$
F_{m p c}^{\kappa}=2 \cdot G \cdot f_{c},
$$

and, at point $\mathrm{A}$, in the flange - inclined face contact, the sliding friction force arises from the axial load per wheel G:

$$
F_{m p c}^{\Gamma}=F_{n} \cdot f_{c}=f_{c} \cdot \frac{G}{\cos \alpha}
$$

We derive the motion equation at the moment of the beginning of the wheelset module inclined face contact

or

$$
m a_{3.1 .1}=m a_{2}-F_{1}-F_{m p c}^{\Gamma}-2 F_{m p c}^{\kappa},
$$

$$
m a_{3.1 .1}=m a_{0}-2 G \cdot f_{\kappa}-4 G \cdot f_{c}-\alpha \cdot V \cdot \sqrt{m_{k n} \cdot C_{r}}-f_{c} \cdot \frac{G}{\cos \alpha}
$$

After the transition of the wheelset from sliding friction to rolling friction, at the wheel flange - inclined face contact, the rolling friction force arises from the axial load G per wheel

$$
F_{m p \kappa}^{\Gamma}=F_{n} \cdot f_{\kappa}=f_{\kappa} \cdot \frac{G}{\cos \alpha},
$$

on the second non-padded wheel from axial load $\mathrm{G}$, a rolling friction force also appears on the wheel thread -rail contact

$$
F_{m p \kappa}^{\kappa}=G \cdot f_{\kappa} .
$$

The equation of motion along the face with this transition takes the form:

$$
m a_{3.1 .2}=m a_{3.1 .1}-F_{m p \kappa}^{\Gamma}-F_{m p \kappa}^{\kappa},
$$

or

$$
m a_{3.1 .2}=m a_{0}-3 G \cdot f_{\kappa}-4 G \cdot f_{c}-\alpha \cdot V \cdot \sqrt{m_{\kappa n} \cdot C_{r}}-f_{c} \cdot \frac{G}{\cos \alpha}-f_{\kappa} \cdot \frac{G}{\cos \alpha}
$$

T3.2 - in this section, the shoe is unloaded, and the wheel flange starts rolling along the level section of the module wedge. In this case, under the action of the axial load between the flange and the wedge, and the wheel tread and the rail, the rolling friction forces FГтрк and Fктрк respectively, will arise, which, pertinently, will be equal, i.e.

$$
F_{m p \kappa}^{\Gamma}+F_{m p \kappa}^{\kappa}=2 G \cdot f_{\kappa} .
$$

The motion equation in this section is

$$
m a_{3.2}=m a_{3.1 .2}-F_{m p \kappa}^{\Gamma}-F_{m p \kappa}^{\kappa},
$$

or

$$
m a_{3.2}=m a_{0}-5 G f_{k}-4 G f_{C}-a V \cdot \sqrt{m_{k n} \cdot C_{r}}-f_{C} \cdot \frac{G}{\cos a}-f_{k} \cdot \frac{G}{\cos a} .
$$


T3.3 - in this section, the shoe is loaded again, and rolling friction transforms into sliding friction.

Since the contact at point A occurs on face 4, which has an inclination in the direction of movement, there will be no impact of the flange on the backband and, therefore, the force $\mathrm{F} 1=0$ and the forces arising in the contact (Fig. 3,b) will be derived from the driving force $F$.

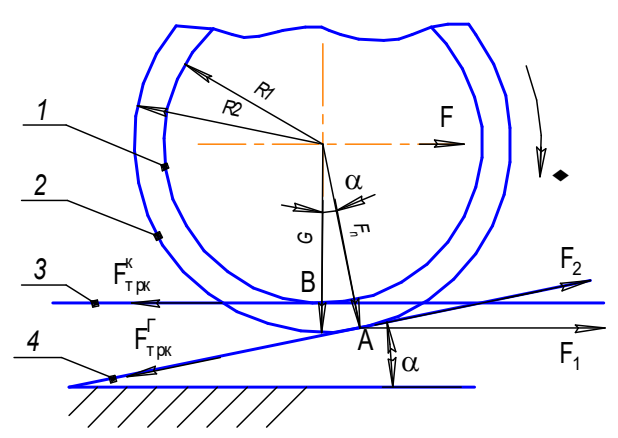

a)

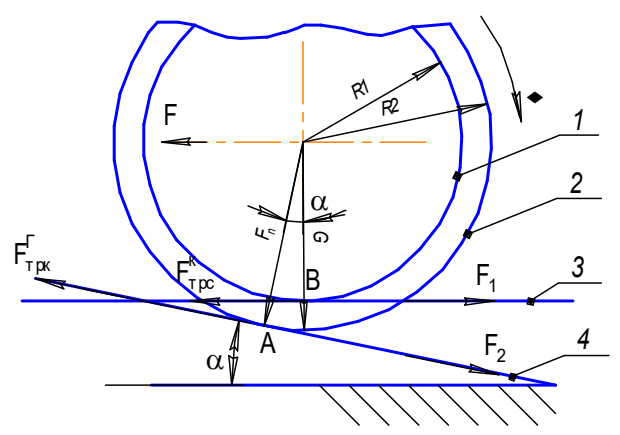

b)

Fig. 3. Wheel - inclined face contact scheme in sections $T_{3.1}$ (a) and $T_{3.3}$ (b) 1 is wheel tread, 2 is flange, 3 is rail, 4 is inclined face of working module

So, at the moment of transition from rolling to sliding at point $\mathrm{A}$, the rolling friction force ГГтрк of the wheel flange along the face

developing into sliding friction force FГтрк will act

$$
F_{m p \kappa}^{\Gamma}=F_{n} \cdot f_{\kappa} \quad F_{m p \kappa}^{\Gamma}=\frac{G}{\cos \alpha} f_{\kappa},
$$

$$
F_{m p c}^{\Gamma}=f_{c} \cdot \frac{G}{\cos \alpha}
$$

At this moment, at point $\mathrm{B}$, the non-padded wheel will be affected by the rolling friction force Fктрк arising between the rail and the wheel surface from the axial load $\mathrm{G}$ per wheel

$$
F_{m p \kappa}^{\kappa}=f_{\kappa} \cdot 2 G
$$

and developing into sliding friction force of the wheel along the rail and skate on the rail

$$
F_{m p c}^{\kappa}=2 G \cdot f_{c}
$$

We compose the motion equation in this section

$$
m a_{3.3 .1}=m a_{3.2}-F_{m p \kappa}^{\kappa}-F_{m p \kappa}^{\Gamma},
$$

or

$$
m a_{3.3 .1}=m a_{0}-6 G \cdot f_{k}-4 G \cdot f_{c}-\alpha \cdot V \cdot \sqrt{m_{k n} \cdot c_{r}}-f_{c} \frac{G}{\cos \alpha}-2 f_{\kappa} \frac{G}{\cos \alpha}
$$

and

$$
m a_{3.3 .2}=m a_{3.3 .1}-F_{m p c}^{\Gamma}-F_{m p c}^{\kappa}
$$

or

$$
m a_{3.3 .2}=m a_{0}-6 G \cdot f_{\kappa}-6 G \cdot f_{c}-\alpha V \cdot \sqrt{m_{k n} \cdot C_{r}}-2 f_{\kappa} \cdot \frac{G}{\cos \alpha}-2 f_{c} \cdot \frac{G}{\cos \alpha}
$$


This sliding will continue in the T4 section, while the sliding friction forces between the brake skate and the rail from the force $\mathrm{G}$ and the sliding friction force in the contact of the non-padded wheel tread and the rail will also act from force G. Since these values will be practically equal, we can write

$$
F_{m p c}^{\kappa}=2 G \cdot f_{c}
$$

and, therefore, the motion equation will have the following form:

$$
m a_{4}=m a_{3.3 .2}-F_{m p c}^{\kappa}
$$

or

$$
m a_{4}=m a_{0}-6 G \cdot f_{k}-8 G \cdot f_{c}-\alpha V \cdot \sqrt{m_{\kappa n} \cdot c_{r}}-2 f_{\kappa} \cdot \frac{G}{\cos \alpha}-2 f_{c} \cdot \frac{G}{\cos \alpha}
$$

The fourth stage (T4). At this stage, as well as at the second one, the wheelset is skidding on the skate brake

$$
m a_{4}=m a_{2}=m a_{0}-2 G \cdot f_{\kappa}-2 G \cdot f_{c} .
$$

The fifth stage (T5). At this stage, passing the derailer, the wheelset is released from the skate brake and starts rolling along the rails, as at the stage $\mathrm{T} 1$, therefore

$$
F_{m p \kappa}^{\kappa}=2 G \cdot f_{\kappa}
$$

We compose the motion equation at the final stage:

or

$$
m a_{5}=m a_{4}-F_{m p \kappa}^{\kappa}
$$

$$
m a_{5}=m a_{0}-8 G \cdot f_{k}-8 G \cdot f_{c}-\alpha V \cdot \sqrt{m_{\kappa n} \cdot c_{r}}-2 f_{\kappa} \cdot \frac{G}{\cos \alpha}-2 f_{c} \cdot \frac{G}{\cos \alpha}
$$

The resulting equation is a complete equation for the movement of rolling stock according to the anti-slide safety system and, therefore, using it, you can calculate the magnitude of the driving force at any stage of movement in accordance with the kinematic cyclogram. Once again, we transform the equation deriving it with respect to the driving force value in the $\mathrm{i}$-th section

$$
m a_{i}=m a_{0}-8 G\left[f_{k}\left(1+\frac{1}{4 \cos \alpha}\right)+f_{c}\left(1+\frac{1}{4 \cos \alpha}\right)\right]-\alpha V \cdot \sqrt{m_{\kappa n} \cdot c_{r}}
$$

Then, we compose the car motion equation for the braking position having $\mathrm{n}$ modules, for example, $\mathrm{n}=2$ (Fig. 4).

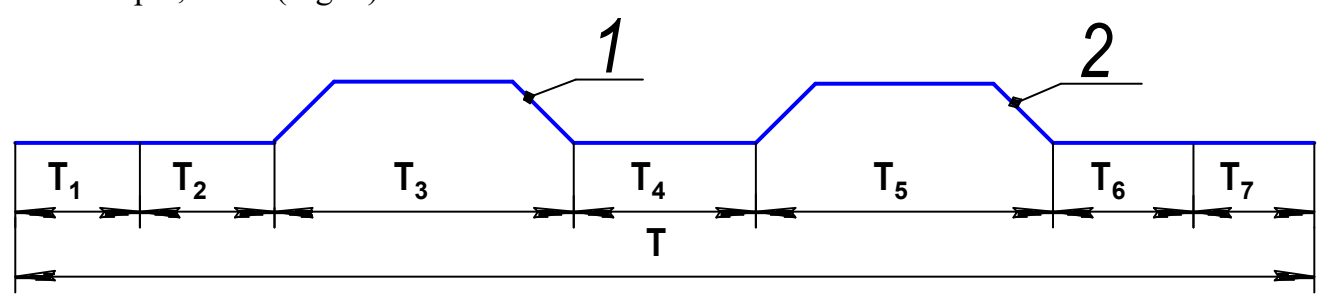

Fig. 4. Cyclogram of car movement on brake position at $n=2.1,2$ are anti-slide safety system modules

We write the driving force variation at each stage through $\Delta \mathrm{F}$. As can be seen from the 
cyclogram, T1,T7 are the sections of wheelset rolling along the rails, respectively, $\Delta \mathrm{F} 1=\Delta \mathrm{F} 7 . \mathrm{T} 2, \mathrm{~T} 4, \mathrm{~T} 6$ are sections of skidding, respectively, $\Delta \mathrm{F} 2=\Delta \mathrm{F} 4=\Delta \mathrm{F} 6 . \mathrm{T} 3, \mathrm{~T} 5$ are sections of the wheel movement modulo, respectively, $\Delta \mathrm{F} 3=\Delta \mathrm{F} 5$. Hence,

$$
m a_{i}=m a_{0}-\Delta F_{1}-\Delta F_{2}-\Delta F_{3}-\Delta F_{4}-\Delta F_{5}-\Delta F_{6}-\Delta F_{7}
$$

The stages $\mathrm{T} 1$ and $\mathrm{T} 7$ are independent of the number of modules, i.e., the driving force variation in these sections is a constant value. The same goes for the stage T6, whereas the number of the stages T2, T4, T3, and T5 will depend on the number of modules.

If the number of modules in the brake position is $\mathrm{n}$, then

$$
m a_{i}=m a_{0}-2 \Delta F_{1}-(n+1) \Delta F_{2}-n \Delta F_{3} .
$$

If the number of brake positions is $\mathrm{s}$, then

$$
m a_{i}=m a_{0}-\left[2 \Delta F_{1}-(n+1) \Delta F_{2}-n \Delta F_{3}\right] \cdot s
$$

As a result of the study [12,13], the equation of the car movement in the "rolling stock hump controller - track" system is obtained, which enables to fit its working elements into the debugged mechanism of the hump with minimum changes.

\section{Discussion and conclusions}

According to the task, a skid adjuster is developed, which eliminates the formation of onesided slides under sorting processes, and an equation is obtained for the car movement in the "rolling stock - hump controller - track" system along the brake positions of the hump yard, which provides fitting the anti-slide safety system, as a new element, into the debugged mechanism of the hump with minimum changes.

The results obtained were used to equip the Voronezh hump yard of the Likhaya station, North Caucasus Railway, with the anti-slide safety system (Fig. 5) [13].

Long-term operation (more than ten years) has shown the reliability of the study which provides the maintenance of the regular splitting up mode with a complete exclusion of the formation of slides.

Currently, the issue of introducing an anti-slide safety system on non-mechanized humps of Severnaya Railway, JSC Russian Railways, is under consideration [13]. 


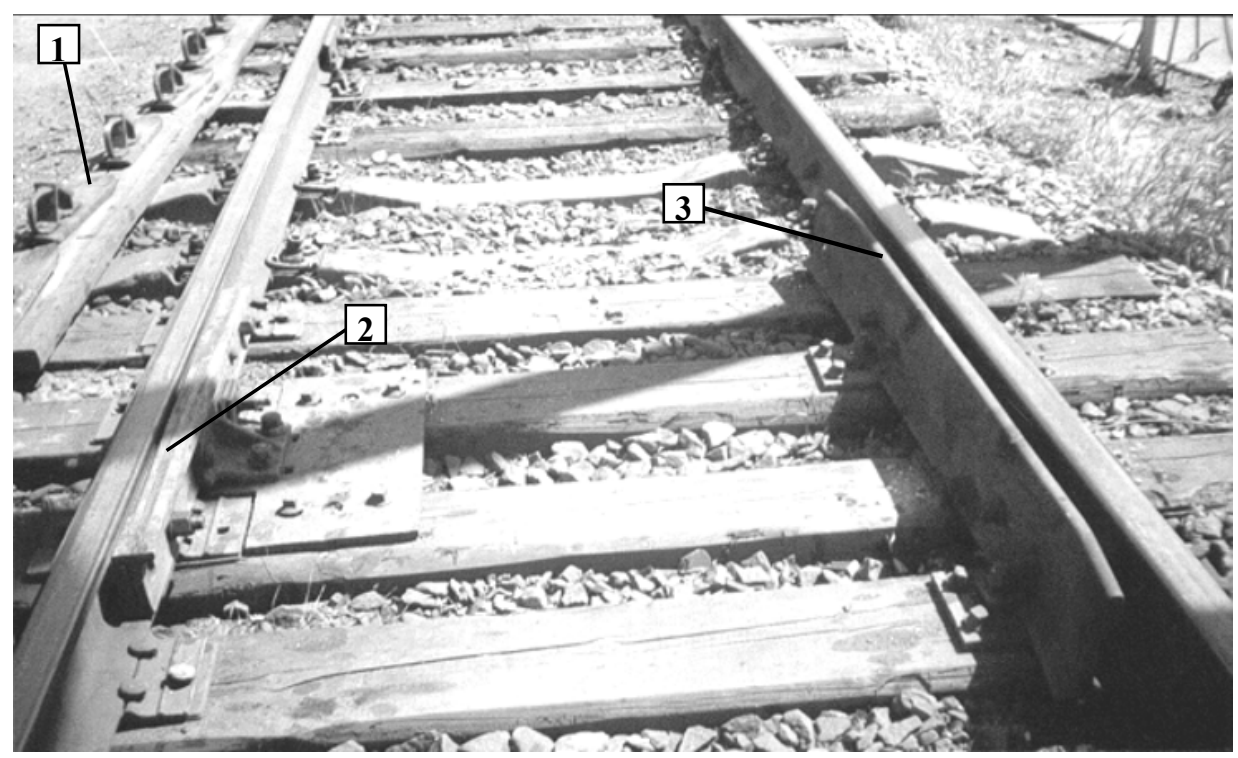

Fig. 5. Picture segment of brake position equipped with ant-slide safety system. 1 is brake skates, 2 is working module, 3 is flange rail

\section{References}

1. V.A. Burakov, Improving train safety by improving and developing station technology (MIIT, 2006). (In Russian).

2. Contact fatigue damage to the wheels of freight cars / Sat. scientific VNIIZHT proceedings (Интекст, 2004). (In Russian).

3. Al.Al. Demyanov, Al.An. Demyanov, V.M. Prikhodko, I.V. Volkov, A.L. Ozyubkin, Modern Science Success. 8, 12, 244-248 (2016).

4. N.N. Chislov, O.N. Chislov, A.D. Petrushin, V.A. Zhukov, Railway transport. 9, 32-35 (2006). (In Russian).

5. I.M. Jarikov, I.V. Gorbunov, A.G. Savickij, A.V. Nikolaev, Railway transport. 1(13), 41-47 (2008). (In Russian).

6. V.T. Kostygov, Friction and lubrication in machines. 4, 10 - 16 (2006). (In Russian).

7. L.C. Guo, W.T. Zhu, L.B. Shi, Q.Y. Liu, Z.B.Cai, W.J.Wang, Wear, 426-427, Part B, 1771-1780 (2019).

8. R. Aghababaei, Wear, 426-427, Part B, 1076-1081 (2019).

9. C.A. Damião, G.C. Alcarria, V.C. Teles, J.D.B. de Mello, W.M. da Silva Jr., Wear, 426-427, Part A, 101-111 (2019).

10. V.G. Yatsenko, Al.Al. Demyanov, Al.An. Demyanov, et al., RF Patent 2321513 (2008).

11. Al.Al. Demyanov, Al.An. Demyanov, et al., RF Patent 2338657 (2008).

12. Al.Al. Demyanov, Al.An. Demyanov, Proc. Int. Sci.-Pract. Conf. "Problems of Mechanical Engineering: Tribology - for Engineering”, 1, 212-216 (2012).

13. Al.Al. Demyanov, Al.An. Demyanov, B.A. Levin, ed., Mashinostroenie, IV - 23, 21 $22(2008)$. 